\title{
Violencia de género, masculinidades y políticas públicas
}

\author{
Graciela Vélez Bautista ${ }^{1}$ \\ Héctor Serrano Barquín ${ }^{2}$
}

Fecha de recepción: 27 de noviembre de 2017

Fecha de aprobación: 3 de abril de 2018

\begin{abstract}
Resumen
El objetivo de este documento es mostrar la importancia que representa analizar la violencia contra las mujeres como un problema complejo, que proviene de la confluencia de diversos factores propios de un sistema social patriarcal, así como enfocar a los hombres y las identidades masculinas como parte central de la problemática y, por lo mismo, de la solución. El proceso metodológico tiene base en el análisis de tres entrevistas semiestructuradas, realizadas a hombres que fueron acusados por sus parejas mujeres de maltrato y violencia hacia ellas. Resulta de interés mostrar que los acusados se consideran inocentes, debido a que, para ellos, es "natural" subyugar a sus esposas.

Los resultados del análisis de esta problemática hacen referencia a la formulación de políticas públicas con enfoque de género, no solo dirigidas a las mujeres, sino a los hombres y al sistema, como una manera adecuada de prevenir, atender y dar soluciones eficientes a disminuir y/o eliminar la violencia contra las mujeres ${ }^{3}$.
\end{abstract}

Palabras clave: masculinidades, políticas públicas, violencia de género.

\section{Gender violence, masculinities and public policies}

\begin{abstract}
This document is aimed at showing how important is to analyze violence against women as a complex problem, which comes from the confluence of
\end{abstract}

1 Doctora en Ciencias Sociales, Coordinadora del Centro de Investigación en Estudios de Género y Equidad e Investigadora de la Facultad de Ciencias Políticas y Sociales de la UAEM, pertenece al Sistema Nacional de Investigadores (SIN-1) Toluca, Estado de México. Contacto: vebag4@yahoo.com.mx

2 Profesor de tiempo completo en la Facultad de Arquitectura y Diseño de la UAEMéx, pertenece al Sistema Nacional de Investigadores (SIN-1) y es líder del CA Contexto Sociocultural del Diseño. Toluca, Estado de México. Contacto: hectorsb2012@yahoo.com.mx

3 El documento que se presenta es parte inicial de una propuesta del proyecto de investigación, denominado "Masculinidad, paternidades y política", en la Universidad Autónoma del Estado de México. 
various factors from a patriarchal social system, as well as from focusing on men and masculine identities as a central part of the problem and, therefore, of the solution. The methodological process is based on the analysis of three semi-structured interviews to men accused for mistreatment and violence by their female partners. It is interesting to show that the defendants see themselves as innocent because they consider "natural" to subjugate their wives.

The analysis of this problem refer to the development of public policies focused on gender -not only oriented to women but also to men and the system - as an adequate way to prevent, attend and give efficient solutions to reduce and/or eliminate violence against women1.

Keywords: Masculinities, public policies, gender violence

\title{
Violência de gênero, masculinidades e políticas públicas
}

\begin{abstract}
Resumo
O objetivo deste documento é mostrar a importância que representa analisar a violência contra as mulheres como um problema complexo, que provém da confluência de diversos fatores próprios de um sistema social patriarcal, assim como colocar o foco nos homens e nas identidades masculinas como parte central da problemática y, pela mesma razão, da solução. O processo metodológico baseia-se na análise de três entrevistas semiestruturadas, realizadas a homens que foram acusados por suas parceiras de maus tratos e violência contra elas. É interessante mostrar que os acusados consideram-se inocentes, devido a que, para eles, é "natural" subjugar às suas esposas.

Os resultados da análise desta problemática referem à formulação de políticas públicas com perspectiva de gênero, não só dirigidas às mulheres, mas também aos homens e ao sistema, como uma maneira adequada de prevenir, atender e dar soluções eficientes a diminuir e/ou eliminar a violência contra as mulheres .
\end{abstract}

Palavras-chave: masculinidades, políticas públicas, violência de gênero

\section{Sumario}

1. Persistencia de la violencia contra las mujeres; 2 . complejidades en la violencia de género; 3. masculinidades y violencia contra las mujeres: ¿una relación necesaria?; hacia una política pública; política y masculinidades hacia la igualdad; reflexiones finales.

\section{Introducción}

Analizar el tema de violencia contra las mujeres en este siglo XXI lleva a cuestionarse acerca de la forma en que es entendida 
por el Estado, por las mujeres y por los hombres en particular, como principales perpetradores de ella, en los más de los casos. ¿Por qué persiste, a pesar de diversas medidas para eliminarla? ¿Cuáles son algunas de sus causas más profundas? ¿En qué medida se ha considerado para su eliminación el estudio de los hombres, la masculinidad y las políticas públicas? Estas cuestiones invitan a reflexionar sobre el sistema sociopolítico de orden patriarcal que privilegia lo masculino sobre lo femenino y que, además, como afirma Amorós (2001; 2008), es un sistema de pactos entre hombres para asegurar su dominio sobre el conjunto de mujeres. El patriarcado como sistema presenta una organización social que otorga mayor poder político y social a los hombres; por ello, los puestos de poder político y militar están mayoritariamente en sus manos. También es importante señalar que es un sistema tradicional que prevalece hasta hoy en la mayoría de las sociedades. Por otra parte, en el sistema patriarcal existen diversas complejidades sociales que se entretejen y propician formas de maltrato y discriminación hacia las mujeres, que por su cotidianidad, costumbre y arraigo social se "naturalizan". Asimismo, es un sistema político porque la toma de decisiones de forma mayoritaria es masculina, ligada al interés de quien ejerce el poder.

\section{La violencia contra las mujeres}

México, en 2007, dio un importante paso en la prevención y atención de la violencia contra las mujeres al promulgar la Ley General de Acceso de las Mujeres a una Vida Libre de Violencia (LGAMVLV, 2007). La creación de esta ley representó, desde luego, la plataforma legal que permitiría proteger el derecho de las mujeres a vivir sin violencia; sin embargo, sabemos que la ley como fundamento para atender este problema es necesaria pero no suficiente. Pese a que su mandato es para todo el territorio nacional, aún no se cuenta con las suficientes estrategias culturales y sociales que permitirían su debida aplicación. Sin duda, esto se debe a la arraigada naturalización de la violencia hacia las muje- 
res $^{4}$, en la que están involucradas ellas mismas y la gran mayoría de la población mexicana. Es decir, que aún se ve como natural que los maridos o parejas masculinas dominen a sus mujeres, que las niñas sean restringidas de ciertos deportes, actividades o trabajos que se consideran propios de los hombres; aun cuando no están escritas tales restricciones, prevalecen en las creencias y valores de una gran parte de la población y se reproducen a través de la educación familiar y hasta escolar. Por ello, "ser mujer" y "ser hombre" en sociedades como la mexicana nos coloca todavía lejos de la igualdad de género.

Al respecto, datos estadísticos como los reportados por la Encuesta Nacional sobre la Dinámica de las Relaciones en los Hogares (ENDIREH, 2016) indican que el 63\% de las mexicanas ha sufrido alguna vez en su vida actos de violencia, tales como abuso sexual, menosprecio verbal, violencia psicológica, física y económica, así como acoso sexual callejero escolar y laboral. En promedio, se estima que durante los últimos tres años (2013 a 2015) fueron asesinadas siete mujeres diariamente en el país. Es decir, todos los tipos y modalidades de la violencia contra las mujeres, incluyendo el feminicidio, siguen en efervescencia, y uno de sus principales fundamentos es la permanencia de creencias y valores tradicionales sobre los estereotipos de género en la sociedad mexicana.

Al respecto, también es importante señalar que en los estudios de caso de los Estados mexicanos en que se ha decretado la Alerta de Violencia de Género contra las Mujeres (AVGM) ${ }^{5}$, que-

\footnotetext{
4 Violencia "naturalizada"contra las mujeres, hace referencia al acostumbramiento y falta de asombro que se da en determinado contexto sobre el maltrato hacia las mujeres, especialmente por los hombres, lo anterior como principio básico del sistema patriarcal.

5 Que a la letra dice: “De conformidad con el artículo 22 de la Ley General de Acceso de las Mujeres a una Vida Libre de Violencia (en adelante, Ley General de Acceso), la alerta de violencia de género contra las mujeres (en adelante, AVGM) es el conjunto de acciones gubernamentales de emergencia para enfrentar y erradicar la violencia feminicida en un territorio determinado, ya sea ejercida por individuos o
} 
da claro que funcionarios/as gubernamentales minimizan las denuncias, las demandas de la sociedad civil, así como tratan de evitar hasta donde pueden que el Grupo Interdisciplinario (GI), que analiza las condiciones que hacen necesario que se dicte la Alerta, determine que así se haga. Pese a que las condiciones del contexto muestren que así debe ser, existe una gran resistencia por parte del funcionariado público encargado de esta acción.

De esta manera, la AVGM representa un mecanismo de actuación de las autoridades públicas que buscan cumplir con las obligaciones del Estado respecto del derecho de las mujeres a una vida libre de violencia, atendiendo específicamente, entre otras, a una de las violaciones más graves a este derecho: la violencia feminicida.

$\mathrm{Su}$ objetivo fundamental es garantizar la seguridad de las mujeres y niñas, a partir del cese de la violencia en su contra, y eliminar las desigualdades producidas por una legislación o política pública que vulnera sus derechos humanos, mediante la determinación de un conjunto de medidas que permitan a las autoridades públicas del territorio cumplir con sus obligaciones en materia de derechos humanos de las mujeres y garantizar su pleno ejercicio.

Sobre este último punto, cabe recordar que la violencia contra las mujeres no solo es feminicida: ésta es su última expresión, pero antes de ella existen una serie de violaciones a los derechos humanos de niñas y mujeres que se dan cotidianamente, como la discriminación laboral ${ }^{6}$ o la estigmatización de niñas y mujeres por practicar deportes de tradición masculina, lo cual obedece a factores culturales, como los estereotipos de género, que confi-

por la propia comunidad estipulada como mecanismo emergente en la Ley General de Acceso de las Mujeres a una Vida Libre de Violencia (2007)".

6 Un clásico ejemplo en México es que en diversas empresas privadas se exige el certificado de ingravidez a las mujeres que solicitan empleo. O el hecho de indicar, por parte del médico de la empresa, que se desvistan completamente para otorgarles el certificado médico. Situación que no sucede con los hombres. 
nan a las mujeres a las actividades domésticas y de cuidado, y a los hombres al mundo del trabajo remunerado y los espacios de reconocimiento social, y que impiden dar las mismas oportunidades de desarrollo a mujeres y hombres.

Es destacable que la violencia "naturalizada" contra las mujeres se acepta en los diversos contextos de México, y cuando hace crisis por alguna ola de feminicidios en lugares determinados, se tiende a dirigir protección hacia la población afectada mediante la creación de organismos de justicia para las mujeres, uno de cuyos problemas es que estos muchas veces son atendidos por personal no especializado en perspectiva de género y justicia, lo cual propicia la atención inadecuada, deficiente e ineficaz hacia las mujeres afectadas; es decir, se toman medidas unilaterales que pretenden atender a las afectadas y se olvida la parte de prevención, fundamental en la lucha contra esta clase de violencia. Refiero lo anterior sobre todo al sistema educativo, que no contempla la perspectiva de género como eje transversal sino, por el contrario, recrea los roles y estereotipos de género mediante juegos y actividades segregadas para niños y niñas.

Por otra parte, los medios de comunicación en México no dimensionan la gravedad de la violencia contra las mujeres, en tanto no informan más que a medias sobre la ocurrencia de casos calificados, como feminicidios, ya sea porque son silenciados o porque se minimiza el problema, dada su naturalización. Cuando se habla de la violencia que es visible porque termina en el feminicidio, se hace un relato del que no se vuelve a hablar para saber en qué terminó el caso; es decir, la mayoría de las veces no se le da seguimiento, a menos que las agrupaciones de la sociedad civil protesten en forma masiva y exijan la investigación. Aun peor, qué pasa con la violencia de género oculta: aquella de la que no se habla, que no es denunciada, pero que se sabe por las encuestas y estadísticas que dan cuenta del maltrato a las mujeres, diversificada en los distintos tipos y modalidades de violencia: esa no es percibida por la sociedad ni se difunde por los medios de comunicación. 
Cabe destacar el impacto de la televisión mexicana como el medio que alcanza una mayor cobertura y que presenta programaciones con alto contenido sexista, porque muestra a las mujeres en el papel femenino tradicional, abocadas exclusivamente al ámbito del hogar y la familia, en el papel que Lagarde (1997) indica como madre-esposa, o como la seductora cuyo único propósito es captar la atención de los hombres y/o conquistarlos, o bien como la mujer santa y moralista al extremo, o la mujer con alta preparación pero que casi siempre está sola, como castigo por no seguir la tradición sino transgredirla. Así también muestra a los hombres manteniendo el dominio y control en todos los ámbitos. Sumisión femenina y dominio masculino es un binomio que propicia la violencia de género y que prevalece en la publicidad y en gran parte de la programación televisiva.

En México, la publicidad comercial que presenta a la mujer cosificada como objeto sexual, expuesta en espectaculares calendarios y carteles, tiene amplia difusión y hasta hoy ni se denuncia ni menos se controla. Aun cuando en ciertos momentos coyunturales se decide el gobierno a contemplar en la agenda pública estrategias de prevención y atención a la violencia contra las mujeres, no se ha considerado importante sugerir o prohibir esta publicidad que denigra a las mujeres y las discrimina como personas. Calleja (2014) explica que en ciertos anuncios se subraya el estereotipo de la mujer como objeto sexual, como cuerpo, invisibilizando su capacidad de independencia y autonomía. Esta publicidad lleva implícita la marca de violencia de género, porque del símbolo es sencillo pasar a la acción.

La violencia simbólica no es menos importante y efectiva que la violencia activa, porque sus efectos son reales en la medida en que permite y sustenta un mundo basado en la asimetría y en las relaciones desiguales entre mujeres y hombres. (p. 23)

En tanto prevalezca este tipo de publicidad comercial y televisiva, sin duda se estará recreando y reproduciendo el control 
y dominio de un género sobre otro, lo cual conlleva la violencia para mantenerlo.

\section{Complejidades en la violencia de género}

Los fenómenos clasificados como "complejos" son así entendidos porque, para ser comprendidos y explicados, fundamentalmente se debe considerar la multiplicidad de factores que los conforman, no solo aludiendo a la suma de los mismos o a su heterogeneidad, sino a la interrelación que presentan. García (2006) lo explica "como la confluencia de múltiples procesos cuyas interrelaciones constituyen la estructura de un sistema que funciona como una totalidad organizada...". En la violencia de género contra las mujeres se encuentran diversas complejidades que han sido poco estudiadas y muchas veces este tipo de violencia se pretende atender de manera simple y aludiendo a uno, dos o tres factores, sin que se haya tenido avances para aminorarla, por lo que es importante analizarla con amplitud, con base en la visibilización de dicha complejidad.

Además de la heterogeneidad, la característica determinante de un sistema complejo es la mutua dependencia de las funciones que dichos elementos cumplen dentro de un sistema total. Esta característica excluye la posibilidad de obtener un análisis de un sistema complejo por la simple adición de estudios sectoriales correspondientes a cada uno de los elementos. (García, 2006, p. 1)

En este sentido, la violencia de género se caracteriza por la concurrencia de una serie de procesos interrelacionados, insertos en la estructura de un sistema que funciona como una totalidad organizada conocida como "sistema patriarcal". En efecto, casi cualquiera supone que lo conoce; sin embargo, hay problemáticas que derivan del mismo, como la violencia contra las mujeres que, como se ha explicado, persiste. Ello obliga a buscar puntos de análisis que han sido menos estudiados para explicarla. Cuando el sistema social la propicia, porque le es inherente como 
parte de un sexismo arraigado, simbólico y activo, que mantiene el vínculo de dominio de lo masculino sobre lo femenino en una especie de complicidad -porque el ejercicio de control de un género sobre el otro permanece naturalizado-, en verdad lleva tiempo deconstruir y reconstruir ese vínculo. En este caso, como indica Izquierdo (2006),

Se hace imprescindible el metaanálisis de las diversas expresiones críticas, de la legislación, de las políticas sociales y de las mismas teorías críticas, ya que los sujetos que las producen son también producto del sexismo que combaten. (p. 242)

Esto explica la visión de muchos jueces y juezas al sentenciar sobre casos de violencia hacia las mujeres; no pueden prescindir de ciertos elementos subjetivos que subyacen en sus juicios.

En este sentido, no es posible aislar la reflexión "ser hombre" y "ser mujer" de las interrelaciones entretejidas en el sistema social para ser uno y otra. Los géneros son constructos estructurales. Cuando los hombres se adscriben a lo masculino y las mujeres a lo femenino, quedan en la posición, condición y situación que su género tiene en la estructura social aunado a sus determinaciones y características personales. (Vélez, 2015, p. 25)

Desde la teoría crítica de género, el sistema patriarcal conlleva en su estructura la discriminación y maltrato hacia las mujeres, su no igualdad social basada en las características de sus cuerpos, hechos para la reproducción y los consecutivos cuidados que ello implica.

En México se ha documentado por la Encuesta Nacional de la Dinámica de los Hogares (ENDIREH, 2016), que el 66,1\% de los 46,5 millones de mujeres de 15 años y más han padecido distintos tipos de violencia: económica, física, sexual y emocional. El lugar más frecuente de violencia hacia las mujeres es la familia; el $38 \%$ ha sido perpetrada por el marido o pareja, así como el $34 \%$ de ellas han sido alguna vez en su vida acosadas, abusadas 
sexualmente y muchas veces violadas. En seguida se encuentra el ámbito comunitario, en el que se documenta que 38\% de mujeres padecieron actos de violencia por parte de desconocidos. Después se indica que, en el ámbito laboral, 26\% de las mujeres han padecido algún acoso sexual o discriminación por ser mujeres, así como menosprecio a sus capacidades con relación a las de los varones. Asimismo un $25 \%$ de las mujeres han padecido acoso escolar de parte de profesores y compañeros.

A pesar de que las cifras documentan solo parte de la realidad, resultan preocupantes; puesto que la violencia se sigue ejerciendo contra las mujeres sin que existan medidas eficaces que la prevengan y detengan, en tanto no puede combatirse solo con acciones o estrategias punitivas, porque en ella intervienen diversos factores que se entrelazan en el sistema sociopatriarcal.

$\mathrm{Al}$ respecto, si en verdad se pretende prevenir, atender y eliminar este tipo de violencia, cabe destacar de qué manera se entretejen diversos factores que propician la violencia contra las mujeres. Como lo explica el modelo ecológico de Uri Bronfenbrenneren, en el que se propone estudiar primero el macrosistema o sistema social, que se configura por los valores culturales y políticos de una sociedad, por la economía y las condiciones sociales. Luego el exosistema, o nivel comunitario, que incluye las redes sociales en que se desarrolla una persona. El mesosistema, referido a las interacciones que se producen entre los microsistemas; y el microsistema, que incluye a la familia $\mathrm{y}$ a los grupos de iguales 
Modelo ecológico de factores asociados con la violencia infligida por la pareja

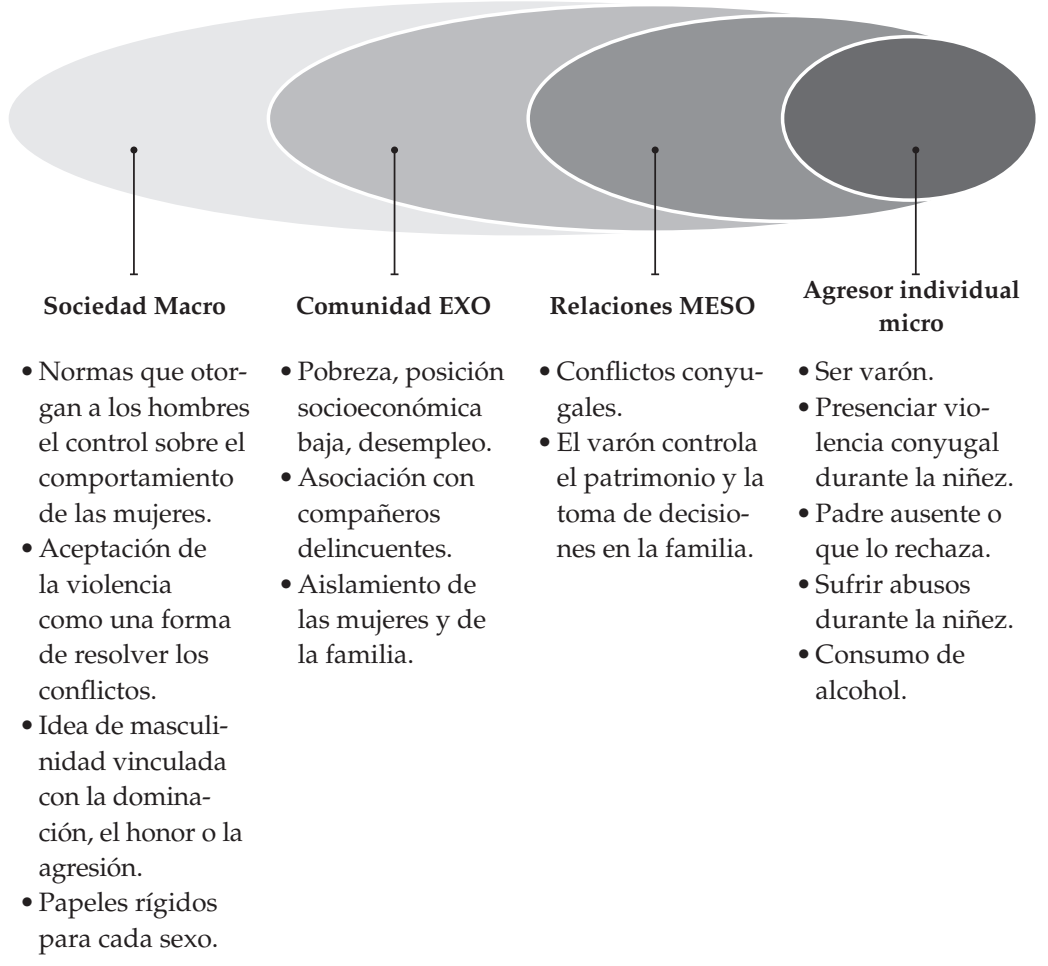

Fuente: Heise, 1997.

Es decir, para comprender de forma íntegra el maltrato a las mujeres es indispensable recurrir a los diversos sistemas en que se desarrolla la persona. Todos ellos interactúan, se intersectan y solo así es posible explicar y entender el origen, sus causas profundas. En ellas habría que establecer líneas de actuación, para transformar el comportamiento de quienes están involucrados.

Sin embargo, las interacciones entre la totalidad y las partes no pueden ser analizadas fraccionando el sistema en un conjunto de áreas parciales, que correspondan al dominio disciplinario de cada uno de los elementos. Sino que corresponde realizar un análisis interdisciplinario, por eso la complejidad de la problemática que representa la violencia de género. 


\section{Masculinidades y violencia contra las mujeres: ¿una relación necesaria?}

Un factor central para analizar la violencia de género es el estudio de las masculinidades. Aunque desde la década de los ochenta el concepto ha permitido superar la designación de los hombres como "patriarcas", enfatiza diferencias a partir de la configuración cultural de las relaciones de poder con las mujeres y entre distintos grupos de hombres. Sin embargo, el uso de la violencia y la agresión va a constituir un rasgo sobresaliente de las identidades de género en los hombres y en las masculinidades.

El estudio de la violencia denominada "intrafamiliar", ejercida por los hombres contra mujeres, niños, niñas y población adulta mayor, parte del reconocimiento de las relaciones desiguales de poder y género. Por ello, el concepto de "violencia masculina" no debe aislarse del entendimiento de los procesos y relaciones sociales de género (Hearn, 1996, p. 29); es decir, no atañe solo a los hombres como individuos, sino que proviene de un sistema complejo que, de no ser analizado como base estructural que a su vez estructura a los sujetos, impediría la transformación de los mismos.

También es posible afirmar que la naturalización de la violencia contra las mujeres en parte se desprende de la educación informal, entendida desde el entorno familiar y desde instituciones como la iglesia donde, de manera permanente, se promueven ideas y creencias que, desde la niñez, fomentan el poder, la fuerza y la posición jerárquica de lo masculino; los padres y abuelos también abonan a este contexto determinista y laxo. Así, tanto la violencia simbólica como la física encuentran en la primera infancia alta permisividad para que los hermanos varones ejerzan sus primeras expresiones de agresividad o violencia hacia las niñas, sin que se penalice o castigue. Después, como adultos, los hombres continuarán ejerciendo su poder ante justificaciones inverosímiles, como las de jueces hombres que en demandas de 
violencia sexual exoneran a violadores con el pueril argumento de que la víctima (generalmente revictimizada) vestía de manera "muy provocativa"; lo insólito de estas justificaciones patriarcales, como lo indica Martínez (2017), es la reciente declaración de un cardenal emérito que, demostrando parte de la visión sesgada de muchos jerarcas católicos ${ }^{7}$ y su feligresía, coincide con la distorsión, resultado de estereotipos históricos y de la forma de vestir de las mujeres, así como por la supuesta "facilidad" con la que ellas son seducidas, según estas declaraciones a los medios. Por otra parte, autores como Rafael Montesinos (2002) plantean la necesidad de "pensar o repensar lo masculino y la masculinidad de otro modo, de tal forma que los hombres 'fuimos empujados' por el movimiento feminista a comenzar a deconstruir (como ya lo había realizado el feminismo) el concepto de lo masculino y la masculinidad". Si bien en la actualidad, a partir de la atención que se da al hombre agresor, sin focalizar solamente en la mujer agredida, se comprende que ni la feminidad ni la masculinidad excluyen al otro género para buscar mejores escenarios de convivencia social.

Surge así la necesidad de fortalecer la reeducación de los hombres, en la cual el estudio de las masculinidades debiera jugar un papel relevante en la visualización de las formas de violencia masculina para, en contraste, fortalecer las masculinidades emergentes, que hablan de factores identitarios más tolerantes, pacíficos y equitativos que, si bien se presentan en pocos casos en la actualidad, debieran alentarse y promoverse como parte de políticas preventivas y educativas, tan necesarias en un país como México, con números inaceptables de feminicidios a la alza. Como explica Bard (2016), nos referimos a las maneras no tradicionales de ser varón, que emergen como respuesta a la confrontación de un contexto de desigualdad, en el que los hombres

Carlos Martínez García. “Misoginia de Juan Sandoval Íñiguez". La Jornada, 22 de noviembre de 2017. Recuperado de: http:/ / www.jornada.unam.mx/2017/11/22/ opinion/022a1pol 
ejercen la dominación mediante la fuerza, el autoritarismo y la violencia.

Actualmente se puede observar que la masculinidad hegemónica ${ }^{8}$ en los sistemas patriarcales recibe el nombre de "machis$\mathrm{mo}^{\prime \prime}$, y éste contiende con otras formas de ser hombre y prácticas más igualitarias de relación entre mujeres y hombres. Justamente, uno de los ámbitos donde se ha cuestionado esta masculinidad es en el doméstico, porque es donde más se da la violencia intrafamiliar.

Como una muestra de la realidad hoy en día, se encuentra que varios de los casos de hombres que han sido denunciados de parte de sus parejas por ejercer violencia de distinto tipo contra ellas, están mostrando su descontento porque, para ellos, "corregir" a sus parejas mujeres o "meterlas en cintura" forma parte de su identidad y hasta de su "deber"; en un ámbito social patriarcal que ha "naturalizado" este comportamiento: es lo que se espera de los hombres; por lo tanto, se sienten juzgados injustamente.

El estudio de casos específicos sobre hombres acusados de violencia por sus parejas permite percatarse de que ellos no reconocen haber ejercido violencia, dada su opinión de que someter a sus mujeres es un "deber" y no un acto agresivo. Es muy probable que no se den cuenta de que sus actitudes verbales y no verbales conllevan agresión, lo cual obedece a la "naturalización" de dicha violencia, como bien lo explica Bonino, a través de los "micromachismos", entendidos como violencia cotidiana naturalizada del orden de lo "micro", al decir de Foucault, de lo capilar, lo casi imperceptible, lo que está en los límites de la evidencia.

\footnotetext{
8 Masculinidad hegemónica, como lo explica Bonino en su escrito "Masculinidad hegemónica e identidad masculina", se refiere al vínculo cultural entre poder y masculinidad, que lleva a los hombres a ser rudos, beligerantes, maltratar a las mujeres, buscar la amistad entre los varones, detestar a los homosexuales, así como despreciar lo femenino.
} 
Para escuchar algunas voces de estos hombres, se realizaron tres entrevistas semiestructuradas a tres hombres de entre cuarenta y cincuenta y cinco años, que pasaron por el proceso de ser acusados por sus parejas de violencia y maltrato hacia ellas. Los aspectos significativos que revelaron son los siguientes:

En mi caso, el juez sólo le daba credibilidad a lo que ella decía, desechó mi testimonio varias veces, y al final le dio la razón, como resultado se me impidió convivir con mis hijas de 14 y 10 años.

Otro de los entrevistados señaló: en el caso de divorcio, los jueces otorgan la guarda y custodia a la madre en tanto hijas e hijos tengan menos de 12 años; lo hacen sin investigar quién es la madre, si los maltrata emocional o físicamente o si tiene problemas de neurosis que perjudican a los infantes. Este es mi caso, ella es una persona inestable emocionalmente. Para demostrarlo presenté el dictamen de un profesional en terapia de pareja, pero el juez nunca lo revisó ni le dio importancia. Acudí con el magistrado presidente, quien me escuchó y al final me dijo: "deja que tu exmujer tenga a tus hijas, tú para qué las quieres, tú ponte a trabajar".

Un entrevistado más señaló: hoy día la justicia favorece a las mujeres, no investigan si dicen verdades o mentiras- mi esposa se niega a que yo conviva con mi hijo. En el juicio pasé la etapa de apelación y ya estoy en la etapa del amparo, espero no perder mi derecho a convivir con mi hijo.

En el primer caso, el acusado siente que el estar ahí es injusto, no es para tanto, considera que su actitud es correcta porque "naturaliza la violencia".

En los dos casos siguientes se conjuntan factores culturales, políticos y subjetivos que dan cuenta de que la costumbre, la socialización de los hombres con el poder y el control les hace sentir que son ellos las víctimas y no los victimarios.

Sin embargo, en los casos mencionados hay razón para señalar que, en efecto, los jueces y juezas parecen considerar que aplicar la perspectiva de género es generalizar el favorecimiento a las 
mujeres en todos los casos, percibiéndolas como "víctimas" y/o "madres buenas" por "naturaleza", y considerar a todos los hombres como "agresores"; unos en potencia y los otros de hecho.

Analizando los tres casos se confirma, en principio, que tanto acusados como juzgadores/as "naturalizan" a las mujeres como "víctimas" y a los hombres como "agresores", sin profundizar en la investigación de cada caso. En seguida, los acusados son incapaces de reconocer sus actos de maltrato y violencia, dado que para ellos dichos actos forman parte del mandato social y de su propia identidad masculina.

La perspectiva de Izquierdo (2006) es ilustrativa, cuando señala que interviene la compulsión social sobre los hombres para que actúen tratando de conservar los privilegios asociados a su virilidad. Ahora bien, cuando se trata de hombres feminicidas la investigación indica que la mayoría se entrega, se suicida o se intenta suicidar, los menos se fugan. Izquierdo señala:

Qué móvil tiene el delito en estos casos? ¿Cumplir con mandatos sociales? La muerte de las mujeres y el posterior suicidio o entrega de quienes las matan adquiere los visos de un acto sacrificial cuando el hombre no logra poner a la mujer en su lugar y por tanto, fracasa en el ejercicio de su poder. De qué otro modo puede interpretarse el hecho de que el 95 por ciento de hombres que asesinan a sus parejas o ex parejas paguen su delito, o sea porque son detenidos, se entregan, se suicidan o lo intentan. (p. 39)

Una explicación más a esta conducta es que, en el proceso de socialización de los hombres con lo masculino, la fuerza es un atributo, de manera que cuando la usan tienden a minimizar su daño, porque en su papel de "hombres" es necesario hacer que se respete su palabra y dominar.

También es notable que hay complicidad en muchos grupos de hombres, cuando en sus conversaciones hacen chistes sobre la inferioridad de las mujeres ante los hombres, o bien cuando se 
burlan de aquellos a los que juzgan como "mandilones", porque participan en el trabajo doméstico o se preocupan mucho por su pareja o familia.

Sin embargo, es posible entender que existe un complemento entre quien agrede y quien es agredida. Ambos responden a los mandatos que su género les ha impuesto y que, a la manera de Bourdieu (2000), se refiere al habitus en el que subyace la subordinación de lo femenino frente a lo masculino.

Por lo tanto, si se desea prevenir, atender y eliminar la violencia de género contra las mujeres, es indispensable aludir al propio sistema social, es decir, a los factores estructurales, culturales y subjetivos del modelo de género; de tal forma que quien agrede para demostrar "hombría" y quien recibe la agresión sumisamente para responder al mandato femenino cambien esa postura $\mathrm{y}$ aspiren al trato igualitario. Lo que no se puede dar solo mediante acciones de castigo para los agresores, y de talleres o pláticas para empoderar a las mujeres. Se podría dar con lo anterior, pero además y sobre todo cuando se enfoque el sistema social y político existente, y desde ahí se generen cambios que impacten el modelo educativo -como uno de los más importantes-, así como una serie de acciones afirmativas en el ámbito económico, laboral y profesional, y también a través de políticas públicas de conciliación entre trabajo remunerado y trabajo familiar.

Es decir, aludiendo a las complejidades de la problemática de la violencia de género, el punto clave es recordar que existe interdependencia de diversos elementos dentro de la totalidad del sistema patriarcal. Esta característica elimina la posibilidad de obtener el análisis apropiado de la violencia de género por la simple suma de estudios correspondientes a cada uno de los elementos que la están provocando; requiere de un tratamiento sistémico con base en políticas públicas que deriven en proyectos y programas permanentes, formulados considerando las causas que la provocan y no solo aumentando sanciones a los agresores y atención a las víctimas en los casos particulares. Es imprescin- 
dible entenderla como un asunto que involucra el propio sistema sociopolítico que prevalece con fundamentos patriarcales.

Lo anterior indica que hace falta investigación de las particularidades de quien acusa y de quien es acusado en cada caso. Además, y como proceso urgente, hace falta que en el sistema judicial se establezca como requisito indispensable que quienes impartan justicia y/o atiendan casos de violencia de género conozcan de manera clara y fehaciente el significado de enfoque de género aplicado al ámbito jurídico y, desde luego, al reciente protocolo para juzgar con perspectiva de género.

De lo contrario, finalmente se perjudica a los infantes. Por ello, resulta de interés enfocar al género masculino en las políticas de igualdad, desde el análisis de la construcción de la masculinidad hegemónica y sus consecuencias hasta las nuevas formas de ser "hombre" y "padre".

\section{Hacia una política pública}

Cabe destacar que, para prevenir y corregir la violencia de género, se hace indispensable aspirar a la igualdad de oportunidades para hombres y mujeres. Desde el modelo ecológico debería implementarse una política estratégica que involucre macrosistema, exosistema, mesosistema y microsistema. Es decir, las acciones de igualdad no solo estarían dirigidas a las conciencias de las personas, hombres y mujeres, sino también a la comunidad, a la ley, a las instituciones y a la sociedad. De otra forma, solo se colocan parches en algunas heridas del mal que conlleva la violencia de género contra las mujeres.

Sin duda, hay asuntos específicos sobre los que se debe incidir, como es el caso de la participación de los hombres en el trabajo doméstico-familiar, el tema de la paternidad plena y los permisos laborales para hombres y mujeres, así como la conciliación entre trabajo y familia y el reconocimiento de tipos de familia distintas a la convencional. Estas medidas son indispensables para apoyar 
la distribución equitativa del trabajo que solo se agradece pero no se paga, y que hasta hoy es el propio de las mujeres.

¿Cuál es el papel del Estado en la promoción de la igualdad de género para prevenir la violencia contra las mujeres? Sin duda su intervención es fundamental; debería ser tema de las agendas pública y de gobierno, ya que debe incorporarse en el sistema educativo, en la comunidad a través de los medios de comunicación, así como en las instituciones públicas y privadas, y desde luego en la familia.

En este sentido, se habla de políticas públicas de igualdad que deben involucrar no solo a las mujeres sino también a los hombres, porque se trata de cambiar las relaciones de género jerarquizadas y sexistas, basadas en estereotipos y roles de género que propician las desigualdades entre mujeres y hombres y que son punto clave para generar violencia y maltrato a quien se considera inferior.

En México, las políticas públicas de igualdad de género que incorporan a los hombres son escasas e incipientes; hasta hoy no se encuentran en la agenda pública, pese a que en las distintas conferencias internacionales sobre la mujer, asambleas y declaraciones internacionales se adoptaron acuerdos para incluir a los hombres en las estrategias hacia la igualdad de género. Es necesario destacar que en 2014 la inclusión de los hombres en las políticas de igualdad fue ratificada en la Declaración de Delhi ( $2^{\text {nd }}$ Men Engage Global Sympsium, 2014). La conformación de políticas públicas internacionales que consideran enfocar a los hombres como aliados hacia la igualdad ha tenido cierta influencia en México, pero aun cuando se ha puesto en la mesa de debate el tema, no se han concretado estas políticas sin las cuales no es posible considerar el logro de la igualdad de género como un cambio necesario para prevenir y eliminar paulatinamente la violencia contra las mujeres. Puesto que los niños y los hombres son parte fundamental del problema, como tales deberían entenderlos y luego llevar a cabo modificaciones que se requieren en distintos espacios y niveles del sistema sociopolítico. 
Fundamentalmente, la agenda pública necesita comprometer al Estado en este propósito, flexibilizar el ámbito laboral e involucrar al entorno familiar.

En este sentido, las políticas públicas consisten en establecer una corresponsabilidad entre gobierno y sociedad, Franco (2013) indica:

Las políticas públicas son acciones de gobierno con objetivos de interés público que surgen de decisiones sustentadas en un proceso de diagnóstico y análisis de factibilidad, para la atención efectiva de problemas públicos específicos, en donde participa la ciudadanía en la definición de problemas y soluciones. (p.18)

En este tenor, una propuesta de políticas públicas con perspectiva de género para los hombres implica de inicio un diagnóstico de la problemática de igualdad de género -que de forma muy escasa a veces incluye a los hombres y solo como factor implicado pero no de forma central-; así que es de capital importancia el diseño de esta política. Con base en un acercamiento a la ciudadanía, al contexto, a la legislación y a las creencias y valores imperantes.

Sin embargo, se sabe que en países como México el presupuesto con perspectiva de género es poco y además se dirige a las mujeres, lo cual es una limitante para ocuparse de los hombres. En este sentido, tendría que haber estrategias que incluyeran a los hombres como factor clave del cambio en las relaciones de género y en la incidencia que tienen en la prevención y eliminación de violencia contra las mujeres. La importancia de ello radica en que, si se considerara como tema prioritario mostrar a la sociedad que existen otras formas de ser hombre, que no exigen mantener control, dominio y poder, se facilitaría la prevención y posterior cambio más igualitario.

\section{Política y masculinidades hacia la igualdad}

De partida, la reproducción de los valores masculinos desigualitarios impide la eliminación de la violencia contra las mu- 
jeres; por ello, se hace necesario promover modelos masculinos alternos que redefinan el "ser hombre", sin olvidar también la redefinición de "ser mujer". Es un asunto de política pública que se ha practicado muy poco y de forma aislada y temporal.

En este caso, se requiere de una estrategia política dirigida a la educación, al mercado de trabajo, a la sociedad y los medios de comunicación, y a la familia; es decir, al nivel micro y macro social que incida en los valores de la niñez y la juventud.

Pero la pregunta es ¿cuáles son los rubros a los que principalmente se tendría que enfocar dicha política pública? Entre algunos fundamentales está el caso de las nuevas paternidades, la conciliación entre trabajo y familia, y sin duda el tema de la salud sexual y reproductiva. No obstante, en este documento se profundiza, solo a manera de ejemplo, en el caso de las nuevas paternidades o paternidades plenas, en tanto cada rubro merece un tratamiento específico.

Al respecto, ¿qué puede decirse del ejercicio de la "paternidad plena"? En principio, que en México se practica poco, pese a los diversos cambios que ha propiciado el adelanto de las mujeres, cuya participación creciente en el ámbito laboral de alguna forma obstaculiza la dedicación completa al cuidado de hijas e hijos y favorece que a veces los padres se involucren en el cuidado y atención de las y los menores. En este sentido, se observa que esta relación cercana y afectiva permite que los hombres se sensibilicen y den rienda suelta a sentimientos de ternura y cuidado. En efecto, los varones expresan poco sus sentimientos, por considerar que eso es propio de lo femenino; sin embargo, cuando deciden o les toca atender a sus infantes la mayoría encuentra el terreno propicio para mostrar su lado afectivo.

Sin embargo, el sistema social, jurídico y político no está preparado para aceptar y marchar acorde con esas formas de ser padre. El sistema judicial, por ejemplo, restringe mucho el derecho de los padres para convivir y obtener la custodia de hijas e hijos, como 
se ha mostrado en el análisis de las entrevistas que forman parte de este documento. Al padre, en la sociedad sexista, se le mira solo como proveedor económico en la mayoría de los casos. Se diría que se borra su participación como cuidador, educador y menos como persona afectiva. De ahí las organizaciones de padres por el derecho a convivir con sus hijos/as. El relato de los entrevistados para este estudio corrobora las afirmaciones anteriores.

Si el gobierno se aboca a diseñar, formular e implementar políticas públicas para varones, considerando los nuevos modelos de ser padre, se ganaría mucho en favor de la igualdad, tanto para los hombres como para las mujeres, y también para hijas e hijos, lo que finalmente incidiría en una mejora en cuanto a la disminución de la violencia de género, porque aumenta la sensibilidad de los hombres y disminuye su agresividad. Burin y Meler (2004) informan que, mediante un experimento, se pudo constatar que cuando a los varones se les encargaban las tareas de cuidado sobre todo con niños/as, se les despertaba cierta sensibilidad que disminuía su agresión.

Al respecto, una política pública contra la violencia de género que incluya a los varones tendría que reunir al menos los siguientes aspectos:

- La participación de los hombres en el cuidado infantil, mediante campañas de información centradas en el papel de los hombres en la vida de las y los infantes, destacando los beneficios que esto conlleva, tanto para niños y niñas como para los padres.

- Promover en las instituciones públicas y privadas el uso de las licencias de paternidad, que propician el acercamiento con el nuevo ser y promueven la participación de ellos en el mundo familiar.

- Publicitar, mediante campañas gubernamentales a través de los medios masivos de comunicación, el rol del padre afectivo no autoritario y dictatorial, pero seguro y consciente del apoyo significativo que posee en la formación de hijos e hijas. 
- Enatizar en la necesidad de políticas sociales que permitan conciliar trabajo con responsabilidades familiares y actividades en el hogar.

- Propiciar una política de género que se dirija a todos los niveles educativos y que incida en el aprendizaje sobre masculinidades y distintas formas de ser hombre y mujer en este contexto.

- Promulgar leyes y nuevos modelos que estimulen la participación de los hombres hacia una paternidad afectiva y más enfocada al cuidado.

- Asimismo, es indispensable implementar leyes que favorezcan la conciliación de la vida laboral y familiar, enfocando desde luego a mujeres y hombres.

Una política contra la violencia de género debe desincentivar la división sexual del trabajo, porque esta promueve la adjudicación del mundo público al hombre y del mundo doméstico a las mujeres, así como la paternidad autoritaria y la idealización de la maternidad.

\section{Reflexión final}

El escrito destaca la importancia de explicar la violencia de género como una problemática integrada en el sistema sociopolítico del patriarcado, prevaleciente en casi todos los contextos y con sus distintas peculiaridades en cada uno.

De manera esencial, enfatiza que muy pocas veces las políticas para atender la violencia de género son adecuadas, más bien se implementa el castigo a los agresores y un programa de apoyo a las víctimas, lo cual solo es un calmante que de forma pasajera proporciona un alivio pero, a la larga, se repite y se reproduce, porque el tratamiento no es el apropiado. En efecto, para lograr un cambio habría que incidir en el sistema social y en todas las expresiones que de él emanan. 
También se destaca la importancia de analizar la socialización de los hombres y sus identidades como parte del sistema, para establecer medidas permanentes y otras pasajeras que permitan eliminar el mal social que representa la violencia de género contra las mujeres.

Así también, es evidente que existe desconocimiento de la perspectiva de género de parte de muchas autoridades, especialmente de jueces y juezas que siguen considerando que solo hay una manera de entender la violencia contra las mujeres: ellas siempre son "víctimas" y ellos siempre son "victimarios". Por lo tanto, importa mucho fortalecer los conocimientos sobre género entre quienes tienen en sus manos impartir justicia.

Es necesario diseñar e implementar políticas públicas con perspectiva de género para los hombres, porque si ellos son parte del problema de la violencia contra las mujeres, deben ser, por lo mismo, parte de la solución.

\section{Referencias}

Amorós, C. (2001). Feminismo, igualdad y diferencia, México: PUEG/ UNAM.

Amorós, C. (2008). Mujeres e imaginarios de la globalización, Argentina: Homo Spiens Ediciones.

Bard, G. (2016). Aferrarse o soltar privilegios de género: sobre masculinidades hegemónicas y disidentes. Revista Península, XI (2) 91-112. UNAM. México.

Bonino, L. Micromachismos: La violencia invisible en la pareja. Recuperado de https://www.joaquimmontaner.net/Saco/dipity_mens/micromachismos_0.pdf 10/04/18.

Bonino, L. Masculinidad hegemónica e identidad masculina. Recuperado de http://www.raco.cat/index.php/DossiersFeministes / article/viewFile/102434/153629 10/04/2018

Bourdieu, P. (2000). La dominación masculina, Barcelona: Anagrama.

Burin, M. y Meler I. (2004). Varones, género y subjetividad masculina. Buenos Aires: Paidós. 
Calleja, J. M. (2014). Informar sobre la violencia contra las mujeres. Guía de buenas prácticas para los medios de comunicación. España: Instituto Aragonés de la Mujer y Universidad de Zaragoza.

Declaración de Delhi (2014). 2nd MenEngage Global Symposium 2014 "Men and Boys for Gender Justice". 10-13 November 2014 India Habitat Centre, New Delhi. Recuperado de http://www. menengagedilli2014.net/uploads/2/4/5/3/24534141/delhi_declaration_and_call_to_action_spanish_final.pdf

Instituto Nacional de Estadísticas y Geografía. (2017). Resultados de la Encuesta Nacional sobre la Dinámica de las Relaciones en los Hogares (ENDIREH) 2016. Recuperado de http://www. inegi.org.mx/saladeprensa/boletines/2017/endireh / endireh2017_08.pdf

Franco, J. (2013). Diseño de Políticas Públicas. Una guía práctica para transformar ideas en proyectos viables, $2^{a}$ edición. México: Editorial IEXE.

García, R. (2001). Interdisciplinariedad y sistemas complejos. Revista latinoamericana de metodología de las ciencias sociales, 1 (1). Buenos Aires. Universidad Nacional de la Plata, FaHCE.

García, R. (2006). Sistemas complejos. Conceptos, métodos y fundamentación epistemológica la investigación interdisciplinaria. Gedisa: España.

Hearn, J. (1996). Men's violence to known women: historical, every day and theoretical constructions by men. En B. Faweett, B. Featherstone, J. Hearn \& Ch. Toft (eds.), Violence and gender relations. Theories and interventions (pp. 22-37). Londres: SAGE.

Heise, L. (1997). La violencia contra la mujer: Organización global para el cambio. En J. L. Edleson y Z. C. Eisikovits, Violencia doméstica: la mujer golpeada y la familia. Barcelona: Garnica.

Inmujeres. (2016). Convención sobre la eliminación de todas las formas de discriminación contra la mujer. Organización de Naciones Unidas. Recuperado de http:/ / www.un.org/womenwatch/ daw/cedaw/text/sconvention.htm

Inmujeres. (2007). Ley General de Acceso de las Mujeres a una Vida Libre de Violencia (2007). Recuperado de https: / / www.gob.mx/ conavim/documentos/ley-general-de-acceso-de-lasmujeres-a-una-vida-libre-de-violencia-pdf

Izquierdo, M. J. (2006). ¿En qué consiste la masculinidad? De lo priva- 
do a lo público, de lo personal a lo racional, de lo psíquico a lo social. En G. Careaga, Debates sobre masculinidades. Poder, desarrollo, políticas públicas y ciudadanía. México. PUEG/ UNAM.

Martínez, C. (2017). Misoginia de Juan Sandoval Iñiguez. Recuperado de http://www.jornada.unam.mx/2017/11/22/ opinion/022a1pol

Montesinos, R. (2002). Las rutas de la masculinidad. Ensayos sobre el cambio cultural y el mundo moderno. Barcelona: Biblioteca Iberoamericana de Pensamiento-Gedisa.

Ramírez, H. (2004). Violencia Masculina en el hogar. Alternativas y soluciones. México: Editorial Pax.

Saucedo, I. (2011). Violencia contra las Mujeres en México. México: PUEG/ UNAM.

Vélez, G. (2015). Violencia de género: escenarios y quehaceres pendientes. México: UAEM. 